\title{
In Silico Evaluation of Radiobiological Hypoxia And Its Effect on Tumour Control During Radiotherapy
}

\author{
Loredana G. Marcu ${ }^{1,2 *}$ and David Marcu ${ }^{2}$ \\ ${ }^{1}$ University of Adelaide, Adelaide, 5000, Australia. \\ ${ }^{2}$ University of Oradea, Oradea, 410087, Romania. \\ *loredana@marcunet.com
}

\begin{abstract}
Modelling in cancer research is a useful tool that can assist in the understanding of tumour growth and treatment response, while also elucidating correlations between various parameters and their interplay that can affect treatment outcome. The aim of the current work was to simulate the growth of a hypoxic head and neck tumour and to treat it with radiotherapy. Tumour hypoxia is one of the main culprits for treatment failure due to the high radioresistance exhibited by the poorly oxygenated cells. The impact of tumour hypoxia on the treatment outcome has been evaluated for the following scenarios: (1) oxygenated versus hypoxic tumour response to radiotherapy; (2) conventional versus hyperfractionated radiotherapy; (3) exponential versus Gaussian distribution of the partial oxygen tension within the hypoxic fraction, and (4) the effect of various hypoxic fractions within the tumour on treatment outcome. The model has shown that hypoxia-related parameters have a strong influence on the tumour's response to irradiation. The results indicate a strong need for pre-treatment quantification of the tumour's hypoxic fraction and its distribution within the tumour in order to assess the effect of hypoxia on treatment outcome and to personalise the treatment for a high therapeutic ratio.
\end{abstract}

\section{Introduction}

Tumour control in radiotherapy is achieved by targeting those cell categories that are able to repopulate the tumour from surviving cells. These cells usually exhibit higher radioresistance due to various factors such as: hypoxia, stem-like properties or intrinsic radioresistance.

Several clinical studies are looking into possible ways to overcome tumour recurrence in aggressive cancers, however with limited success. While clinical trials are important prerequisites in establishing novel treatment techniques and schedules, they also have several shortcomings. Trials are lengthy processes that involve several determining factors for their success, and often the clinical outcome involves unexpected side effects. Perhaps one of the most important downsides is the impossibility to forecast the outcome with certainty.
In silico or computational modelling has lately become an important tool in medical sciences that is able to offer predictive assessments of complex processes that dictate radiotherapy outcome. The noninvasive approach and the possibility for quantitative treatment optimisation using in silico simulations confer this tool an important position among traditional methods of treatment evaluation.

One of the main factors responsible for treatment failure in radiotherapy is the hypoxic content of the tumour population that leads to radioresistance. Oxygen is regarded as the most effective sensitizer during radiotherapy. When ionizing radiation interacts with the tissue, free radicals are produced as a result of ionization. In the presence of oxygen the hydroxyl radical produced by water radiolysis is oxidized and creates an unrepairable lesion to the DNA strand. On the other hand, during hypoxia the free 
radicals are commonly reduced by sulfhydryl compounds thus the DNA damage is either scarce or easily repaired. Clinical hypoxia is quantified via the partial oxygen tension $\left(\mathrm{pO}_{2}\right)$, which was shown to have very low values for hypoxic tumours (below $10 \mathrm{mmHg}$ ).

Due to tumour heterogeneity and wide variations in the hypoxic content among patients, patients enrolled in clinical trials should undergo a thorough selection for the outcome to be clinically significant and reliable. Modelling the variation of hypoxia and the interplay with other kinetic factors related to tumour development offers an easier way to simulate different scenarios and to evaluate the impact of each individual parameter on treatment outcome.

The aim of the present work was to develop a virtual tumour having biologically valid parameters and to assess the tumour's response to radiotherapy as a function of kinetic parameters and radiobiological hypoxia.

\section{Methods}

Given that tumours originating from different tissues present with different kinetics and dynamics, the current model has focused on one tumour group, namely head and neck cancers that originate from the squamous epithelium.

The virtual tumour growth follows the evolution of a biologically realistic head and neck cancer, by encompassing all those kinetic parameters that are responsible for tumour development: cell lineage (cell types), percentages of various cell types, cell cycle length/duration, apoptotic cell death, and volume doubling time [1]. The major cell types that are incorporated in the tumour are:

- Tumour initiating stem cells with unlimited proliferating ability

- Differentiated cells with limited proliferative ability

- Quiescent cells, without the ability to proliferate (unless a stimulus, such as irradiation, triggers them from the quiescent state into the cell cycle).

Hypoxia is an important parameter that has been employed by the current model. The hypoxic fraction varies from $10 \%$ to $50 \%$ of the tumour population and is classified into three categories: mild hypoxia (i.e. $5 \mathrm{mmHg}<\mathrm{pO}_{2}<10 \mathrm{mmHg}$ ), moderate hypoxia $\left(2.5 \mathrm{mmHg}<\mathrm{pO}_{2}<5\right.$ $\mathrm{mmHg})$ and severe hypoxia $\left(\mathrm{pO}_{2}<2.5\right.$ $\mathrm{mmHg}$ ). Two distributions of the hypoxic fraction as a function of $\mathrm{pO}_{2}$ values have been modeled (see figures 1 and 2).

The effect of fractionated radiotherapy has been modeled using the Linear Quadratic formalism of cell survival:

$$
S=\exp \left[-\left(\alpha \mathrm{D}+\beta \mathrm{D}^{2}\right)\right](1)
$$

where S represents the surviving cells, D is the overall radiation dose and the $\alpha$ and $\beta$ parameters are tumour specific parameters that characterize radiosensitivity. Based on the scientific literature for head and neck cancer, a surviving fraction after a radiation dose of 2 Gy $\left(\mathrm{SF}_{2}\right)$ of $54 \%$ has been implemented [3].

Using the linear quadratic relationship of cell survival (1) and considering the value for the $\alpha / \beta=8$ [3] surviving fractions for altered fractionation radiotherapy (i.e. less than 2 Gy dose per fraction) have been determined and implemented in the model.

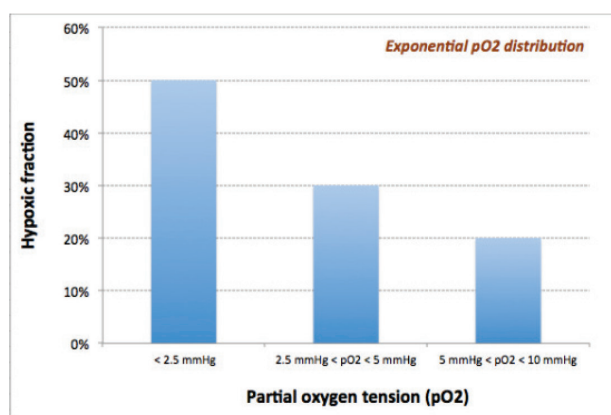

Figure 1. Exponential distribution of hypoxic fraction in the virtual tumour. 


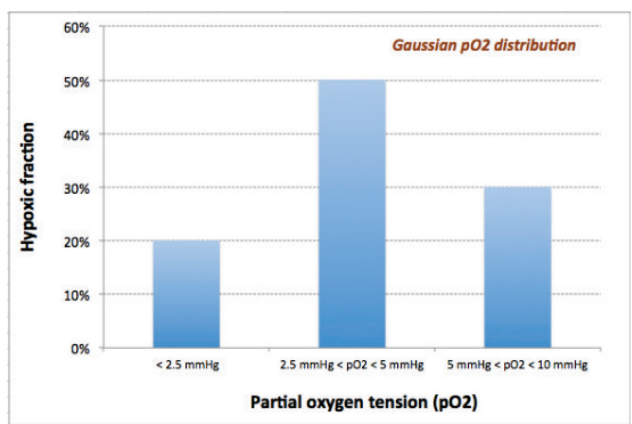

Figure 2. Gaussian distribution of hypoxic fraction in the virtual tumour.

Using the linear quadratic relationship of cell survival (1) and considering the value for the $\alpha / \beta=8$ [3] surviving fractions for altered fractionation radiotherapy (i.e. less than 2 Gy dose per fraction) have been determined and implemented in the model.

The impact of tumour hypoxia on the treatment outcome has been evaluated for the following scenarios:

(1) Oxygenated versus hypoxic tumour response to radiotherapy;

(2) Conventional versus hyperfractionated radiotherapy;

(3) Exponential distribution of $\mathrm{pO}_{2}$ versus Gaussian distribution, and

(4) The effect of various hypoxic fractions (10\%-30\%-50\%) on tumour control.

\section{Results}

Based on the parameters described in the Methods section, the following results have been obtained:

\section{Oxygenation status}

The fact that the best radiosensitising agent is oxygen is illustrated in figure 3, where the same hyperfractionated radiotherapy schedule has been simulated on a hypoxic tumour $(10 \%$ hypoxic fraction) and on an oxygenated tumour, respectively.

While the oxygenated tumour can be controlled by radiotherapy, hypoxia is shown to dictate radioresistance.

\section{Radiotherapy schedule}

The virtual tumour has been treated with both conventional radiotherapy ( 2 Gy a day,
5 days a week over 7 weeks) and hyperfractionated radiotherapy (1.2 Gy twice daily, 5 days a week, over 7 weeks). The cell survival curves represented by the active hypoxic cell population (figure 4) show that the hypoxic tumour cannot be controlled with conventional fractionation, as there is still a significant percent of active hypoxic cells that survived irradiation.

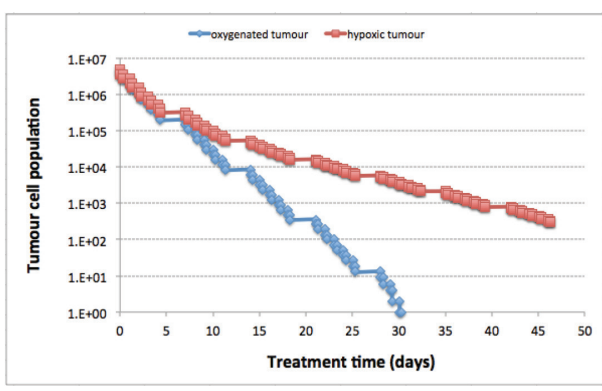

Figure 3. Simulation of oxygenated versus hypoxic tumour response to radiotherapy

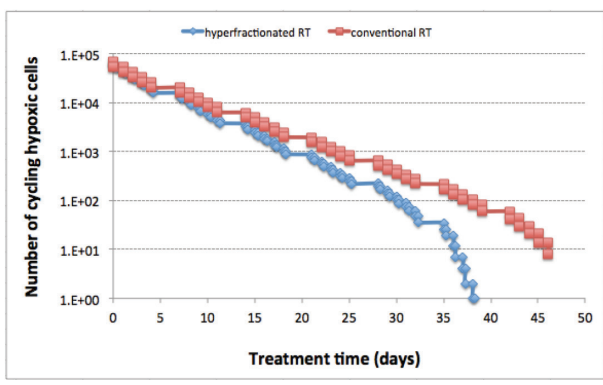

Figure 4. Simulation of conventional and hyperfractionated radiotherapy on a virtual head and neck tumour with hypoxic fraction.

\section{$\mathrm{pO}_{2}$ distribution}

Exponential as well as Gaussian distribution of the hypoxic fraction as a function of $\mathrm{pO}_{2}$ have been simulated during hyperfractionated radiotherapy (figure 5). Since tumours present with various distributions of the partial oxygen tension, patients having similar tumour kinetics but different $\mathrm{pO}_{2}$ distributions within the tumour will respond differently to the same treatment schedule. Large population of severely hypoxic cells (such as in the 
exponential distribution) lead to a more aggressive tumour than other distribution patterns.

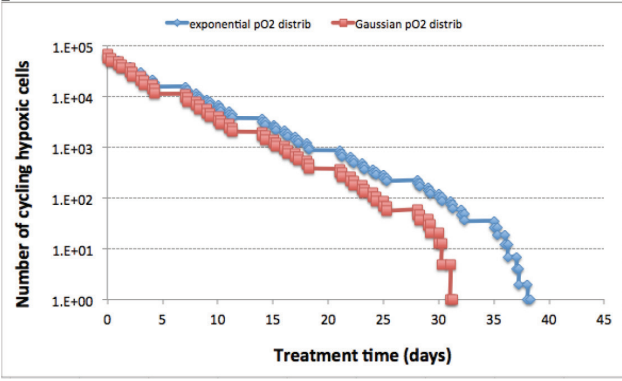

Figure 5. The effect of exponential versus Gaussian distribution of $\mathrm{pO}_{2}$ on tumor response to treatment.

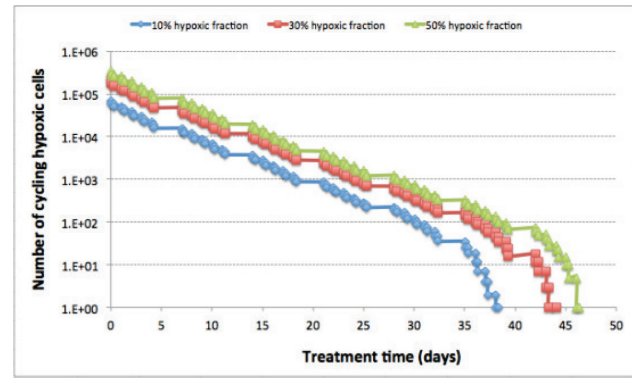

Figure 6. The effect of the hypoxic fraction on tumour response to treatment.

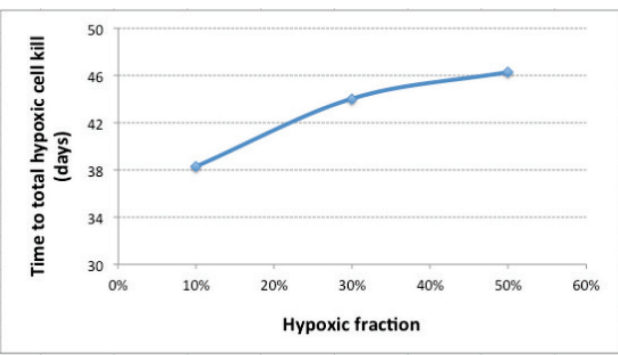

Figure 7. The relationship between the hypoxic fraction and tumour control (expressed as the time to total hypoxic cell kill).

\section{Hypoxic fraction}

Three hypoxic fractions have been implemented in the virtual tumour growth: $10 \%, 30 \%$ and $50 \%$. Figure 6 illustrated the survival curves of the active hypoxic population in the three situations, when all the other parameters are kept constant. The $\mathrm{pO}_{2}$ distribution is considered exponential and the tumour is treated with hyperfractionated radiotherapy. Figure 7 shows a supralinear relationship between the hypoxic fraction and tumour control.

\section{Conclusions}

A virtual head and neck tumour with radiobiological hypoxia has been developed using Monte Carlo modelling techniques. The in silico model has shown that hypoxiarelated parameters have a strong influence on the tumour's response to irradiation. Conventionally fractionated radiotherapy is not effective on hypoxic tumours as the surviving cells can regrow the tumour. Hyperfractionated radiotherapy is recommended for hypoxic head and neck cancers as multiple and smaller doses a day, with an overall larger total dose (84 Gy versus 70 Gy) can overcome tumour resistance to radiation. Nevertheless, it is crucial to undertake a pre-treatment quantification of the tumour's hypoxic fraction and its distribution within the tumour in order to assess the effect of hypoxia on treatment outcome and to personalise the treatment for a high therapeutic ratio.

\section{Acknowledgment}

This work was supported by a grant of the Ministry of National Education, CNCSUEFISCDI, Project no. PN-II-ID-PCE2012-4-0067.

\section{References}

1) L. Marcu, T van Doorn, I. Olver and S. Zavgorodni, Australas. Phys. Eng. Sci. Med 25 (2002) 155.

2) I. Tannock and R. Hill, The basic science of oncology, 3rd edition (McGraw-Hill, New York, 1998) 357392.

3) S. Bentzen and M. Baumann, The linear-quadratic model in clinical practice, in Basic clinical radiobiology, 3rd edition, editor G.G. Steel (Arnold Publisher, London, 2002) 134-146. 